\title{
Improving Argos Doppler location using multiple-model smoothing
}

\author{
Rémy Lopez ${ }^{1 *}$, Jean-Pierre Malardé ${ }^{1}$, Patrick Danès ${ }^{2,3}$ and Philippe Gaspar ${ }^{1}$
}

\begin{abstract}
Background: Argos is a dedicated system for geo-localization and data collection of platform terminal transmitters (PTTs). The system exploits a constellation of polar-orbiting satellites recording the messages transmitted by the PTTs. The localization processing takes advantage of the Doppler effect on the carrier frequency of messages received by the satellites to estimate platform locations. It was recently demonstrated that the use of an Interacting Multiple Model (IMM) filter significantly increases the Argos location accuracy compared to the simple Least Square adjustment technique that had been used from the beginning of the Argos localization service in 1978. The accuracy gain is especially large in cases when the localization is performed from a small number of messages $(n \leq 3)$. The present paper shows how it is possible to further improve the Argos location accuracy if a processing delay is accepted. The improvement is obtained using a fixed-interval multiple-model smoothing technique.

Results: The location accuracy of the smoother is evaluated with a data set including over 200 platforms equipped with an Argos transmitter and a GPS receiver, providing the ground truth. The use of the smoother reduces the platforms' location error. On average, compared with the IMM filter, the smoother achieves an error reduction of about one-third for locations based on two or three messages. For one-message locations, the error is typically divided by two.

Conclusion: The smoother proves to reduce the platforms' location error compared to the IMM filter. The error reduction is all the more significant as the number of messages involved in the location is small. This new processing technique targets Argos applications with a limited emitting power or operating in difficult environmental conditions, such as wildlife tracking, for which obtaining more accurate locations is more important than obtaining locations in real-time.
\end{abstract}

Keywords: Argos system, Doppler location, Multiple-model smoothing, Rauch-Tung-Striebel formulae, Target tracking

\section{Background}

The Argos system has been used since 1978 for geolocalization and data collection of platform terminal transmitters (PTTs) in the fields of wildlife tracking, oceanography and maritime safety. The system is based on a constellation of low-altitude polar-orbiting satellites, which record messages transmitted by the PTTs in a dedicated bandwidth around the $401.650-\mathrm{MHz}$ frequency. The Argos localization exploits the Doppler

\footnotetext{
*Correspondence: rlopez@cls.fr

${ }^{1}$ Collecte Localisation Satellites, 11 rue Hermès, 31520 Ramonville

Saint-Agne, France

Full list of author information is available at the end of the article
}

shift on the carrier frequency of the messages, induced by the relative motion of the satellite and the platform. When a message is received by a satellite, the shifted carrier frequency is measured before being transmitted to the Argos processing centers via a network of ground stations. All frequency measurements acquired during a satellite pass over a platform are used to estimate the platform's position.

Between 1978 and 2011, a classical non-linear Least Squares (LS) estimation technique was used to compute Argos positions. In March 2011, an Interacting Multiple Model (IMM) filter was implemented in the Argos operational processing center [1] and is now the nominal algorithm used by Argos for platforms' localization 
in real-time. This new method reduces the Argos positioning error compared to the LS estimates. The error reduction is especially large when less than four messages are received during a satellite pass, a situation mostly encountered with small, low output power transmitters used in difficult environmental conditions (dense forests, rough seas...). Such transmitters are mostly used for animal tracking. The IMM filter also systematically provides a characterization of the positioning error (which was not the case with the LS positioning algorithm) and increases the amount of locations delivered to Argos users [1].

The IMM filter computes locations recursively by combining the frequency measurements of a satellite pass with a set of $M$ realistic prior dynamics and observation models [2]. In practice, the IMM handles a bank of $M$ unscented Kalman filters (KF) [3, 4] to adapt the dynamics model to the active platform behavior or mode (a random walk or a directed movement for Argos). Filtering assimilates past and present frequency measurements to estimate positions. This is an adequate approach when information is needed in real-time, such as in fishing vessel monitoring systems. However, many Argos applications, such as wildlife tracking, do not usually require information provision in real-time. In that case, a Kalman smoother can be used instead of a filter [5]. Filters are indeed devised to make use of measurements acquired before and at the estimation time while smoothers also use subsequent observations. This means that each location can be inferred with a greater amount of information, and thus a better accuracy can be achieved, at the cost of a delayed estimation.

This paper is the direct sequel of [1] in which the LS estimation technique previously used for Argos positioning was compared to the IMM filter. The comparison was performed on a large data set obtained from over 200 mobiles carrying both an Argos transmitter and a GPS receiver used as ground truth. After a brief reminder of the Argos Doppler positioning problem and its solution based on an IMM filter, the new smoothing method is presented and its performances are evaluated using the same data set as [1].

\section{Methods}

Notations are standard: $P(\cdot), p(\cdot)$ and $E[\cdot]$ represent a probability, a probability density function (pdf) and an expectation, respectively. $N(. ; \bar{x}, P)$ stands for the real Gaussian distribution function with mean $\bar{x}$ and covariance $P$. The transpose operator is denoted by ${ }^{~}{ }^{\mathrm{T}}$.

\section{Multiple-model filtering}

Each Argos localization is computed from the set of $n_{k}$ frequency measurements recorded during the satellite pass $k=0,1, \ldots$ (the time index) over a platform. The processing estimates the longitude $\lambda_{k}$, the latitude $\varphi_{k}$ and the transmitting frequency $f_{t, k}$ of the platform at the average date $t_{k}$ of the measurements. These unknowns are assumed invariant during the satellite pass, which lasts at most $15 \mathrm{~min}$. For the $l$ th measurement of the satellite pass $k$, the received frequency $f_{r,(k, l)}$ is linked to these unknowns by the Doppler observation function $\mathcal{H}_{(k, l)}$ :

$$
\begin{aligned}
f_{r,(k, l)} & =\mathcal{H}_{(k, l)}\left(\lambda_{k}, \varphi_{k}, f_{t, k}\right) \\
& =f_{t, k}\left(1-\frac{\dot{r}_{(k, l)}\left(\lambda_{k}, \varphi_{k}\right)}{c}\right)+v_{(k, l),}
\end{aligned}
$$

where $c$ is the speed of light and $v_{(k, l)}$ is a zero-mean Gaussian noise modeling measurement uncertainties. The quantity $\dot{r}_{(k, l)}$ is the relative radial velocity between the platform and the satellite at the reception time of the associated message. In the following, the vector $\left(\lambda_{k}, \varphi_{k}, f_{t, k}\right)^{T}$ is denoted by $x_{k}$ and the vector $\left[f_{r,(k, 1)}, \ldots, f_{r,\left(k, n_{k}\right)}\right]^{T}$ of measurements by $z_{k}$.

The vector $x_{k}$ is then considered as the state vector of a stochastic dynamical system described by $M$ possible prior dynamic models and one observation model. This multiple-model framework is also called a "jump Markov system" or "Markovian switching system" [6]. The discrete index $m_{k} \in \mathcal{M}$ represents the active mode or model (i.e., the platform behavior) during the sampling period $\left(t_{k-1}, t_{k}\right]$. The sequence of modes $m_{0}, m_{1}, \ldots$ is a homogeneous finite-state Markov chain whose transition probabilities $P\left(m_{k}=i \mid m_{k-1}=j\right)=\pi_{j i}$ are known for all $j, i$. The event $m_{k}=i$ will henceforth be noted $m_{k}^{i}$. At initial time, the prior state pdf writes as the Gaussian mixture

$$
p\left(x_{0}\right)=\sum_{i \in \mathcal{M}} p\left(x_{0} \mid m_{0}^{i}\right) P\left(m_{0}^{i}\right),
$$

where the pdfs $p\left(x_{0} \mid m_{0}^{i}\right)=\mathcal{N}\left(x_{0} ; \hat{x}_{0 \mid 0}^{i}, P_{0 \mid 0}^{i}\right)$ and the probabilities $P\left(m_{0}^{i}\right)$ are given for all modes $i$. The state dynamics between $k-1$ and $k$ conditionally on the active mode $i$ over $\left(t_{k-1}, t_{k}\right]$ is described by the state space equation

$x_{k}=g_{k-1}^{i}\left(x_{k-1}\right)+w_{k-1}^{i}, \quad w_{k-1}^{i} \sim \mathcal{N}\left(w_{k-1}^{i} ; \bar{w}_{k-1}^{i}, \mathcal{Q}_{k-1}^{i}\right)$,

where the dynamics noise $w_{k-1}^{i}$ is white and independent of $x_{0}$ and where the sequence $\left\{g^{i}, \bar{w}_{.}^{i}, Q_{.}^{i}\right\}_{i \in \mathcal{M}}$ is given. Under the same assumption that mode $i$ is active, the measurement $z_{k}$ is related to the state vector by the output equation

$$
z_{k}=h_{k}^{i}\left(x_{k}\right)+v_{k}^{i}, \quad v_{k}^{i} \sim \mathcal{N}\left(v_{k}^{i} ; \bar{v}_{k}^{i}, R_{k}^{i}\right),
$$

where the observation noise $v_{k}^{i}$ is white and independent of $x_{0}$ and $\left\{w_{l}^{i}\right\}_{l \in\{1, \ldots, k-1\}}$, and where the sequence $\left\{h^{i}, \bar{v}^{i}, R_{.}^{i}\right\}_{i \in \mathcal{M}}$ is given. For Argos localization [1], the transitions between 
modes are assumed equiprobable. The admissible prior dynamics of the platform are a random walk and a biased random walk, and the output equations boil down to $h_{k}^{i}\left(x_{k}\right)=h_{k}\left(x_{k}\right)=\left[\mathcal{H}_{(\mathrm{k}, 1)}\left(x_{k}\right), \ldots, \mathcal{H}_{\left(\mathrm{k}, \mathrm{n}_{\mathrm{k}}\right)}\left(x_{k}\right)\right]^{T} \forall i \in \mathcal{M}$.

For a given platform, filtering aims at determining recursively for $k=0,1, \ldots$ the posterior pdf $p\left(x_{k} \mid z_{1: k}\right)$ of the state vector $x_{k}$ conditioned on the past and present measurements $z_{1: k}=\left(z_{1}, \ldots, z_{k}\right)$. The exact posterior pdf $p\left(x_{k} \mid z_{1: k}\right)$ in the considered multiple-model context writes as a Gaussian mixture with a number of terms growing geometrically with the time index $k$ [7]:

$$
\begin{aligned}
p\left(x_{k} \mid z_{1: k}\right)= & \sum_{\substack{i_{0: k} \in \mathcal{M}^{\mathrm{k}+1}\\
}} p\left(x_{k} \mid m_{0: k}=i_{0: k}, z_{1: k}\right) \\
& \times P\left(m_{0: k}=i_{0: k} \mid z_{1: k}\right)
\end{aligned}
$$

The IMM filter recursively approximates this pdf by maintaining a fixed number of terms in the mixture so that

$$
p\left(x_{k} \mid z_{1: k}\right) \approx \sum_{i \in \mathcal{M}} \tilde{p}\left(x_{k} \mid m_{k}^{i}, z_{1: k}\right) P\left(m_{k}^{i} \mid z_{1: k}\right),
$$

where $\tilde{p}\left(x_{k} \mid m_{k}^{i}, z_{1: k}\right)=\mathcal{N}\left(x_{k} ; \hat{x}_{k \mid k}^{i}, P_{k \mid k}^{i}\right)$. For output purposes, the posterior $p\left(x_{k} \mid z_{1: k}\right)$ can then be approximated by the moment-matched Gaussian pdf $\mathcal{N}\left(x_{k} ; \hat{x}_{k \mid k}, P_{k \mid k}\right)$ with mean $\hat{x}_{k \mid k}=E\left[x_{k} \mid z_{1: k}\right]$ (the location estimate) and covariance $P_{k \mid k}=E\left[\left(x_{k}-\hat{x}_{k \mid k}\right)\left(x_{k}-\hat{x}_{k \mid k}\right)^{T} \mid z_{1: k}\right]$ (the location error covariance).

\section{Multiple-model smoothing}

We are interested in obtaining the smoothed Argos locations within a fixed interval $0 \leq k \leq T$ of satellite passes. The integer $T$ is the terminal time index of the interval or, equivalently, the last satellite pass of the emitting period of the PTT. The aim is to compute the first two moments $\hat{x}_{k \mid T}=E\left[x_{k} \mid z_{1: T}\right]$ and $\quad P_{k \mid T}=E\left[\left(x_{k}-\hat{x}_{k \mid T}\right)\left(x_{k}-\hat{x}_{k \mid T}\right)^{T} \mid z_{1: T}\right]$ of the smoothed state density $p\left(x_{k} \mid z_{1: T}\right)$ of the satellite pass $k$ conditioned on all the frequency measurements $z_{1: T}$ over the fixed interval ending at $T$.

In the single-model case, two approaches are generally considered to build the smoothed mean and covariance. The first solution, called the "two-filter smoother", combines the posterior mean and covariance computed from a classical forward-time Kalman filter with the predicted mean and covariance produced by a backward-time filter initialized at time $T$ with no information [8]. Another mathematically equivalent solution is the Rauch-TungStriebel smoother that runs a conventional forward-time Kalman filter until time $T$, then smartly recombines the forward-time moments into the smoothed estimates inside a backward recursion [9]. This recursion is straightforwardly initialized with the forward-time moments at time $T$.

The multiple-model smoothing problem can be solved with similar schemes although the smoothed state density still writes as an exponentially growing mixture:

$$
\begin{aligned}
p\left(x_{k} \mid z_{1: T}\right)= & \sum_{\substack{i_{0: k} \in \mathcal{M}^{k+1}\\
}} p\left(x_{k} \mid m_{0: k}=i_{0: k}, z_{1: T}\right) \\
& \times P\left(m_{0: k}=i_{0: k} \mid z_{1: T}\right) .
\end{aligned}
$$

The method proposed in [10] fuses the estimates of two IMM filters, propagating forward and backward in time, respectively. Again, the backward filter must be initialized without prior information and, for Argos, this amount to perform a LS adjustment with a minimum of four measurements [1]. In some configurations (PTTs with low battery levels or damaged antenna at the end of the emitting period), this event may hardly occur as most of satellite passes contains very few messages. A forward filtering pass followed by a backward smoothing pass (without initialization) is then more suitable to handle such tricky cases. References [11-13] presented a backward pass using the statistics computed by a Generalized Pseudo-Bayesian of order 2 (GPB2) filter. The GPB2 filter [14] is a multiple-model filter displaying a similar accuracy to the IMM strategy but at the higher cost of running $M^{2}$ Kalman filters in parallel. Similarly, Ref. [15] exploits the statistics of a custom multiple-model filter (with $M^{2}$ Kalman filter predictions and $M$ Kalman filter updates). References $[16,17]$ proposed more recently a backward scheme exploiting only the statistics computed from a genuine IMM. This solution has been eventually chosen for the Argos localization processing to retain the good performances and the low computational cost of the IMM filter. With this method, the smoothed density writes as a mixture of $M$ Gaussian pdfs

$$
p\left(x_{k} \mid z_{1: T}\right) \approx \sum_{i \in \mathcal{M}} \tilde{p}\left(x_{k} \mid m_{k}^{i}, z_{1: T}\right) P\left(m_{k}^{i} \mid z_{1: T}\right),
$$

where $\tilde{p}\left(x_{k} \mid m_{k}^{i}, z_{1: T}\right)=\mathcal{N}\left(x_{k} ; \hat{x}_{k \mid T}^{i}, P_{k \mid T}^{i}\right)$. Again, a moment-matched approximation can lead to the smoothed location estimate $\hat{x}_{k \mid T}$ and the associated smoothed location error covariance $P_{k \mid T}$.

\section{Test data set}

The multiple-model smoother was tested as in [1] on a set of 228 Argos PTTs carrying an Argos transmitter and a GPS receiver. The data set includes 60 birds (marabou storks and geese), 23 terrestrial animals (blue wildebeests and bighorns), 78 marine animals (flatback turtles, green 
turtles, Galapagos sea lions and elephant seals), 44 drifting buoys and 23 ships. A total of 162,503 Argos locations were computed and 430,370 GPS positions were collected via the Argos uplink (see details in Table 1). The GPS locations are considered as the ground truth in this study as their worst case error is around $100 \mathrm{~m}$.

\section{Results and discussion}

As the number of messages collected during a satellite pass influences the location accuracy, the distribution of locations w.r.t. the number of recorded messages within the satellite pass is shown in Fig. 1. Interestingly, marabou, bighorn and wildebeest display very few satellite passes with less than four messages. These platforms are generally located in clear areas with a low ambient noise. Moreover, they transmit every 3 or 5 days during a few hours with a short repetition period between consecutive messages, thereby increasing the average number of messages received per pass. Geese platforms are also cycled but they migrate in Asia where the noise floor is higher [1], sometimes reducing the message reception rate. Drifters and ships emit at high power without interruption and in generally favorable transmission conditions (unobstructed horizon). More than three messages per satellite pass are thus usually available to compute their positions. Locations with three messages or less only occur for (short) satellite passes at low elevation angles. On the opposite, marine animals generally carry small, low-power transmitters operating in difficult conditions near the possibly rough sea surface. In addition the surface time is often limited so that these animals must most of the time be located from 1, 2 or 3 messages only.

Figure 2a displays the means and standard deviations of the location error for the smoother and the IMM filter when more than three messages per pass are available to estimate a position. These mean errors and standard deviations are computed relative to reference GPS ground truth as described in [1]. For such locations obtained with a large number of messages, smoothing yields mean location errors that are 3-20\% smaller than those obtained with the IMM filter. The largest mean error reductions are obtained for the elephant seals (20\%), the flatback turtles (15\%) and the drifters $(14 \%)$. The reduction of the standard deviation ranges from $7 \%$ (marabou, goose, blue wildebeest and ship) to $25 \%$ (elephant seal). For two- or three-message locations, the situation is even better (Fig. 2b): the mean error associated with the smoothed position estimates is, on average, reduced by $33 \%$ compared to the filtered case. The

Table 1 List and characteristics of the PTTs included in the dataset

\begin{tabular}{|c|c|c|c|c|}
\hline \multirow[t]{2}{*}{ Type of mobile } & \multirow{2}{*}{$\begin{array}{l}\text { Number of plat } \\
\text { forms }\end{array}$} & \multicolumn{2}{|c|}{-Number of locations } & \multirow[t]{2}{*}{ Data owner and references } \\
\hline & & Argos & GPS & \\
\hline $\begin{array}{l}\text { Marabou stork (Leptoptilos } \\
\text { crumeniferus) }\end{array}$ & 5 & 3759 & 8250 & Neil and Liz Baker (Tanzania Bird Atlas, P.O. Box 1605, Iringa, Tanzania) \\
\hline Goose (Anser indicus) & 55 & 21,220 & 80,980 & $\begin{array}{l}\text { Lucy Hawkes (University of Exeter, Penryn Campus, Cornwall, TR10 9EZ, } \\
\text { UK), Charles Bishop (Bangor University, Bangor, Gwynedd, LL57 2DG, } \\
\text { UK) and Pat Butler (University of Birmingham, Birmingham, B15 2TT, UK) } \\
\text { [18] }\end{array}$ \\
\hline $\begin{array}{l}\text { Blue wildebeest (Conno- } \\
\text { chaetes taurinus) }\end{array}$ & 10 & 2180 & 4144 & Moses Selebatso (Western Kgalagadi Conservation Corridor Project) \\
\hline Bighorn (Ovis canadensis) & 13 & 2159 & 1704 & $\begin{array}{l}\text { Norv Dallin (Nevada Department of Wildlife, Eastern Region, } 60 \text { Youth } \\
\text { Center Drive, } 89801 \text { Elko, NV, USA) }\end{array}$ \\
\hline $\begin{array}{l}\text { Flatback turtle (Natator } \\
\text { depressus) }\end{array}$ & 19 & 24,205 & 21,809 & $\begin{array}{l}\text { Kellie Pendoley (Pendoley Environmental Pty Ltd, 2/1 Aldous Place, } \\
\text { Booragoon, WA 6154) }\end{array}$ \\
\hline $\begin{array}{l}\text { Green turtle (Chelonia } \\
\text { mydas) }\end{array}$ & 24 & 15,959 & 13,340 & $\begin{array}{l}\text { Simon Benhamou (Centre d'Ecologie Fonctionnelle et Evolutive, U.M.R. } \\
5175 \text { Montpellier, France) [19] }\end{array}$ \\
\hline $\begin{array}{l}\text { Galapagos sea lion (Zalo- } \\
\text { phus wollebaeki) }\end{array}$ & 9 & 1680 & 3027 & $\begin{array}{l}\text { Daniel Costa (Department of Ecology and Evolutionary Biology Institute } \\
\text { of Marine Sciences, Long Marine Lab University of California, Santa Cruz } \\
\text { Santa Cruz, California, USA) [20] }\end{array}$ \\
\hline $\begin{array}{l}\text { Elephant seal (Mirounga } \\
\text { angustirostris, Mirounga } \\
\text { leonina) }\end{array}$ & 26 & 13,120 & 62,664 & $\begin{array}{l}\text { Daniel Costa [21] } \\
\text { Christophe Guinet (Centre d'Etudes Biologiques de Chizé, } 79360 \text { Villiers- } \\
\text { en-Bois, France) }[22,23]\end{array}$ \\
\hline Ship & 23 & 23,404 & 36,425 & Various \\
\hline Drifter & 44 & 54,817 & 175,633 & $\begin{array}{l}\text { Luca Centurioni (Scripps Institution of Oceanography, Physical Oceanog- } \\
\text { raphy Research Division, } 9500 \text { Gilman Drive, Mail Code 0213, La Jolla, } \\
\text { CA, } 92093 \text { USA) }\end{array}$ \\
\hline Total & 228 & 162,503 & 430,370 & \\
\hline
\end{tabular}




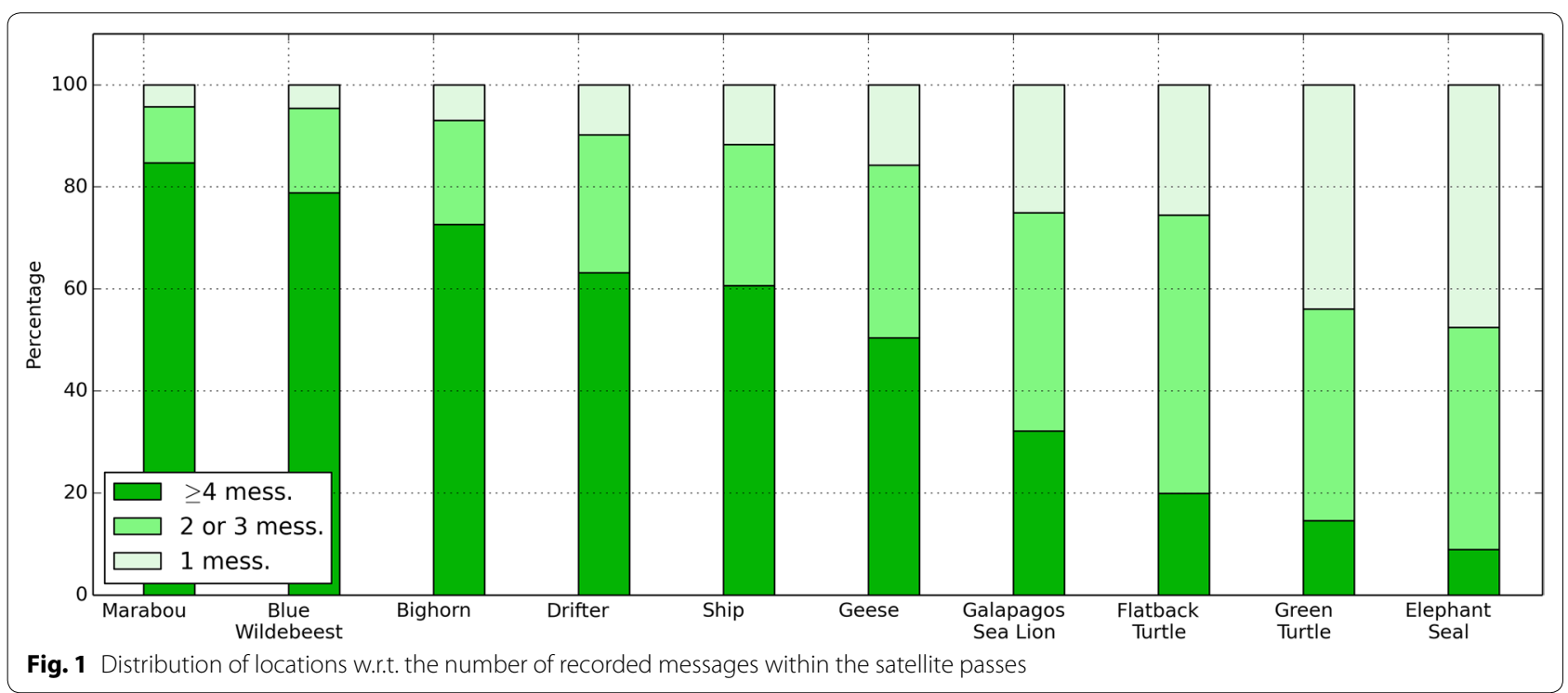

decrease in the standard deviation is between $31 \%$ (goose and gnu) and $68 \%$ (bighorn). The gain obtained with the smoother is the most important with one-message locations (Fig. 2c): the mean error for the smoothed positions is about half of that for the filtered positions, and the standard deviations are reduced from $32 \%$ (marabou and goose) to $77 \%$ (bighorn). The mean error is as small as $550 \mathrm{~m}$ for drifters and remains below $7 \mathrm{~km}$ in the worst case (goose).

Broadly speaking, the location accuracy is largely influenced by the observation geometry of the satellite pass. The most favorable observation geometry-or equivalently the best location accuracy-is obtained when

C1: the platform is located from $5^{\circ}$ to $15^{\circ}$ left or right of the sub-satellite track (using the Earth centered angular distance),

C2: messages are numerous and uniformly distributed within the satellite pass (the platform is observed under multiple angles by the satellite).

Both conditions are actually randomly fulfilled as the latter depends on the emitting environment (radio masks, industrial noise...) and the former on the relative position between the platform and the satellite. The smoother has a positive impact on the location accuracy when $\mathrm{C} 1$ is not satisfied. Figure 3 displays for the drifters the average location error versus the Earth-centered distance to the sub-satellite track (all locations are retained). The LS adjustment, the IMM filter and the smoother are compared and the minimum location error is obtained around $7^{\circ}$ of distance for all algorithms. With the LS adjustment and the filter, the error diverges when the platform is on the sub-satellite track $\left(0^{\circ}\right)$ or at the limit of the visibility circle $\left(25^{\circ}\right)$. This behavior is largely less marked with the smoother as the error varies less with the sub-satellite track distance, especially when the platform is located outside the distance interval $\left[5^{\circ}, 15^{\circ}\right]$. The information brought by future Doppler frequency measurements complements the observation geometry in such unfavorable configurations. The results of the previous section also show that the smoother is more efficient if the number of received messages is $\operatorname{low}^{1}$ ( $\mathrm{C} 2$ is not met). The prior dynamics, which brings additional information from the previous satellite pass through the predicted location, improves the pass geometry. This was also observed when comparing the IMM filter to the LS adjustment in [1].

As an example, the trajectory of a migrating elephant seal (Mirounga leonina) is shown in Fig. 4. The animal performed a round trip of nearly $5000 \mathrm{~km}$ between November 2010 and February 2011 from its rookery located on the Kerguelen Islands. Its migration is estimated with on average two messages per pass and 12 passes per day. This example already benefited from the IMM filter [1]. It can be seen that the smoother further increases the congruence between the GPS track and the Argos locations. The filter trajectory displays abnormal peaks associated with one-message locations which are notably improved by the smoother. The improvements apply both when the animal follows a directed motion (Fig. 4b) and when the movement is closer to a random walk before turning back (Fig. 4c).

\footnotetext{
${ }^{1}$ Note that, for a given repetition period of the platform, a lower number of received messages means also they are likely to be unevenly distributed within the satellite pass.
} 

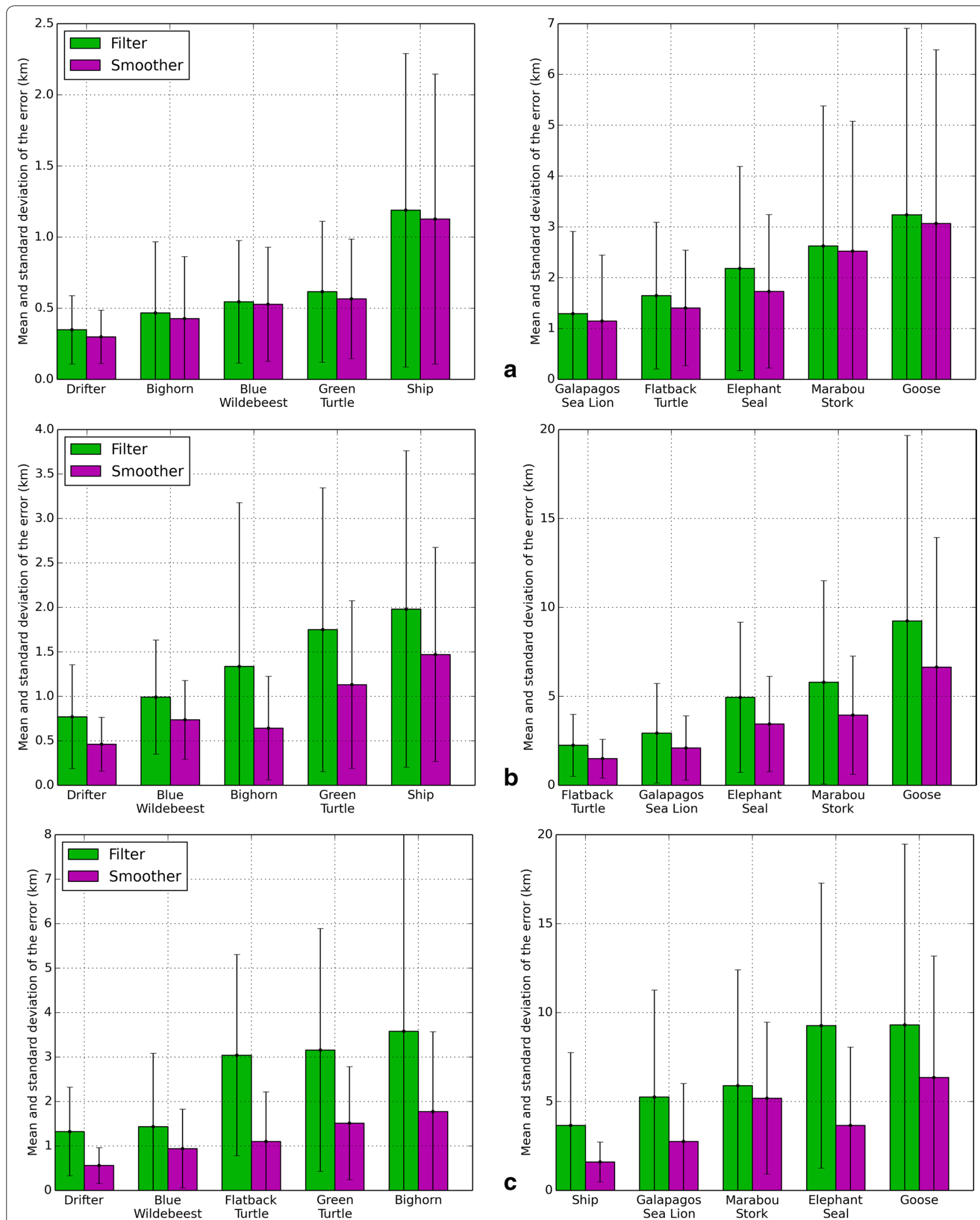

Fig. 2 Mean and standard deviation of the errors; a with at least four messages, $\mathbf{b}$ with two or three messages and $\mathbf{c}$ with one message. Note that, in each figure, the platform types are ordered (from left to right) by increasing IMM filter average errors. The scale of the $y$ axis is changed accordingly (always wider on the right panel) 


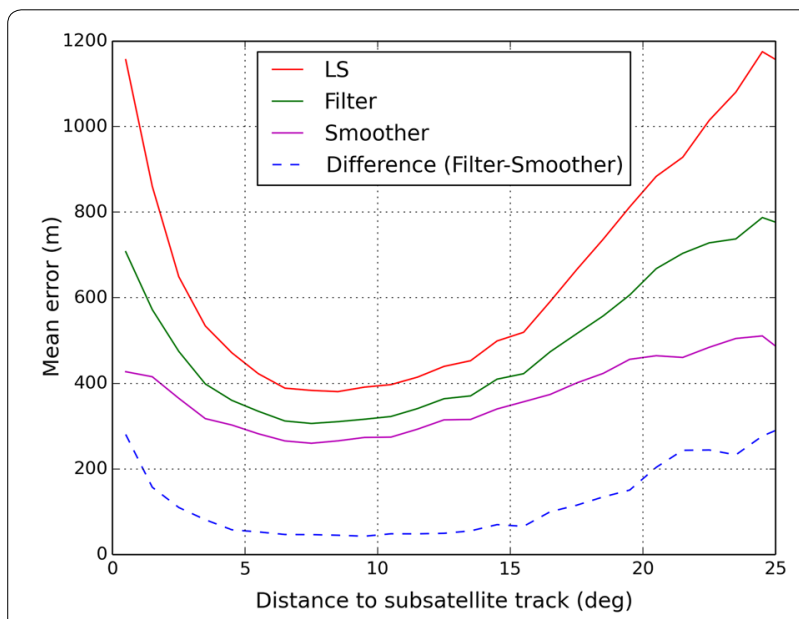

Fig. 3 Mean location error w.r.t. the distance of the platform to the subsatellite track

A moderated "lag" effect was observed on the trajectories produced by the IMM filter, affecting mainly onemessage locations [1]. Table 2 presents for the IMM filter and the smoother the signed average error projected along the trajectory divided by the average error modulus (see [1] for a complete description of the computation). A negative value may indicate a systematic lag with respect to the true location along the trajectory, and a positive value may indicate an advance. For the filter, the ratios are generally negatively signed especially for one-message locations. This is not the case with the smoother as the ratios decrease and are no more systematically negatively signed. Outliers are still observed for blue wildebeests and marabou ( $-36 \%$ and $+71 \%$, respectively) but the amount of one-message locations is too small to compute reliable statistics for both data sets. To better understand why this phenomenon disappears with the smoother, it is worth remembering that the smoothed locations are equivalently produced by fusing the estimates of two filters propagating forward and backward in time. Both filters would be affected by opposite lag effects so that the effect of the forward filter would be compensated by that of the backward filter after the fusion. The cancellation of the lag effect also contributes to the decrease of the location error with one-message passes.

To close the location error analysis, the consistency of the smoother was evaluated by considering as in [1] the $1 \sigma, \sqrt{2} \sigma$ and $3 \sigma$ estimated confidence ellipses. These must theoretically contain 39.3, 63.2 and $98.9 \%$ of the computed locations, respectively. For the LS and IMM algorithms, the percentages of locations falling within the errors ellipses were lower than their theoretical values. Table 3 displays this quantity for the smoother and for the IMM filter. While the smoother still underestimates
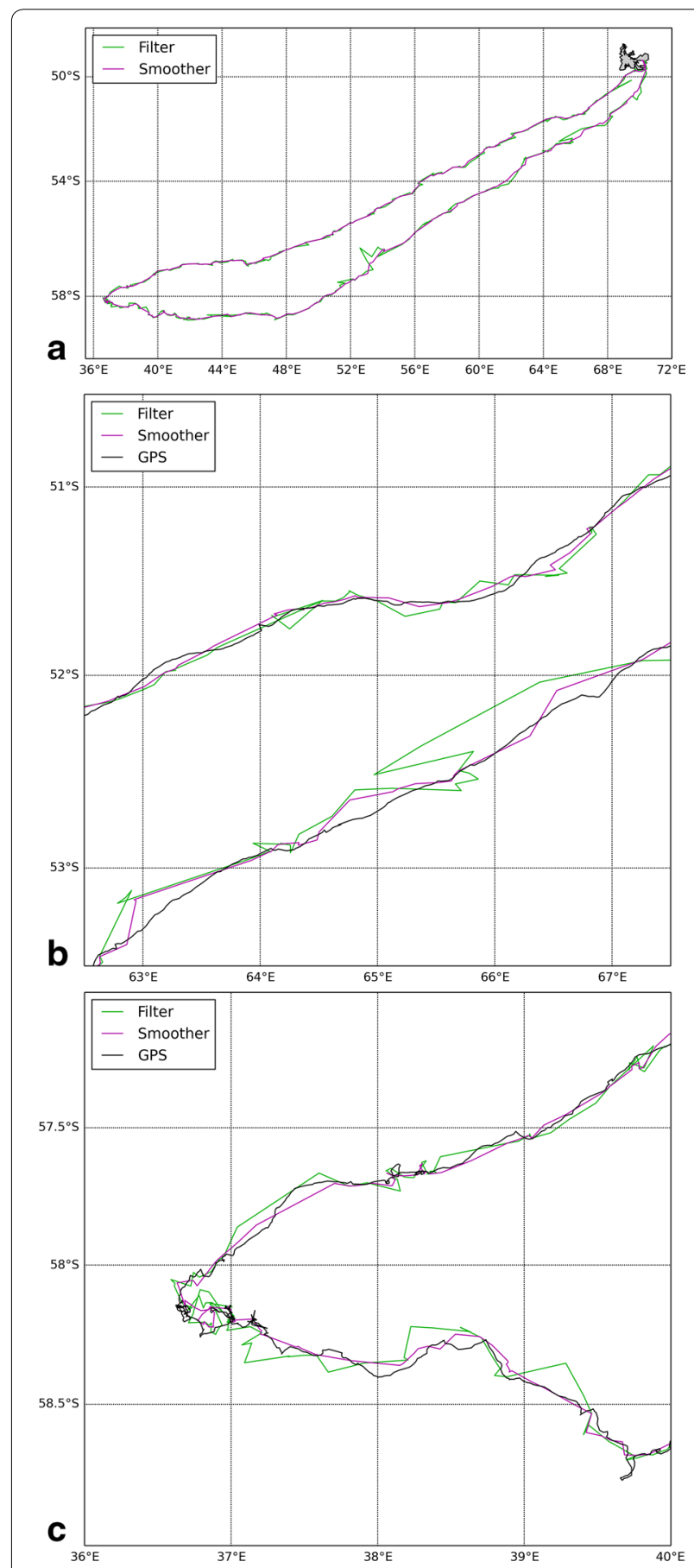

Fig. 4 Comparison of elephant seal tracks between GPS, IMM filter and multiple-model smoother; $\mathbf{a}$ overall trajectory. $\mathbf{b}$ and $\mathbf{c}$ Extracts of the middle and the end of the trajectory, respectively

the actual positioning error, the percentages are on average greater with this new method. In other words, the smoother offers a better characterization of the estimated error than the IMM filter. 
Table 2 Ratio of the signed tangential error on the error modulus

\begin{tabular}{|c|c|c|c|c|c|c|}
\hline \multirow[t]{2}{*}{ Type of mobile } & \multicolumn{2}{|c|}{4 mess. or more } & \multicolumn{2}{|c|}{2 and 3 mess. } & \multicolumn{2}{|l|}{1 mess. } \\
\hline & Filter (\%) & Smoother (\%) & Filter (\%) & Smoother (\%) & Filter (\%) & Smoother (\%) \\
\hline Marabou & 1 & 1 & 10 & 10 & 37 & 71 \\
\hline Goose & 4 & 4 & -1 & -2 & -8 & -1 \\
\hline Blue wildebeest & 3 & 2 & -16 & -11 & -25 & -36 \\
\hline Bighorn & -2 & -1 & 7 & 12 & -19 & 9 \\
\hline Flatback turtle & -13 & -5 & -9 & 10 & -14 & 11 \\
\hline Green turtle & 5 & 16 & -15 & 15 & -22 & 9 \\
\hline Galapagos sea lion & 15 & 17 & -4 & 18 & -2 & 6 \\
\hline Elephant seal & -2 & 13 & -8 & 17 & -18 & 13 \\
\hline Ship & -1 & 1 & -18 & 0 & -24 & -6 \\
\hline Drifter & -14 & 1 & -38 & 8 & -59 & 9 \\
\hline
\end{tabular}

Table 3 Probabilities that computed locations fall within the error confidence ellipses (headers contain the theoretical values)

\begin{tabular}{|c|c|c|c|c|c|c|}
\hline \multirow[t]{2}{*}{ Type of mobile } & \multicolumn{2}{|c|}{$1 \sigma(39.3 \%)$} & \multicolumn{2}{|c|}{$\sqrt{2} \sigma(63.2 \%)$} & \multicolumn{2}{|c|}{$3 \sigma(98.9 \%)$} \\
\hline & Filter (\%) & Smoother (\%) & Filter (\%) & Smoother (\%) & Filter (\%) & Smoother (\%) \\
\hline Marabou & 15 & 14 & 22 & 23 & 51 & 51 \\
\hline Goose & 20 & 20 & 27 & 28 & 49 & 50 \\
\hline Blue wildebeest & 21 & 21 & 34 & 34 & 70 & 70 \\
\hline Bighorn & 33 & 34 & 45 & 47 & 70 & 72 \\
\hline Flatback turtle & 25 & 28 & 37 & 40 & 65 & 68 \\
\hline Green turtle & 28 & 32 & 41 & 45 & 67 & 71 \\
\hline Galapagos sea lion & 22 & 24 & 34 & 35 & 60 & 61 \\
\hline Elephant seal & 18 & 20 & 26 & 29 & 50 & 54 \\
\hline Ship & 21 & 22 & 31 & 33 & 59 & 61 \\
\hline Drifter & 30 & 34 & 45 & 50 & 80 & 84 \\
\hline
\end{tabular}

\section{Conclusion}

Smoothing is a deferred-time processing that assimilates all frequency measurements within the platform emitting period to estimate each point of the associated trajectory. The multiple-model smoother proved to be a new step forward in enhancing the overall quality of the Argos tracks. Compared to the IMM filter, average location errors are indeed reduced by one-third with two or three messages and by half with a single message. The standard deviations of the error also decrease similarly. The smoother displays more uniform performances regardless of the observation geometry associated to the satellite pass, particularly when the PTT is close to the sub-satellite track or at the edge of the satellite visibility circle. Moreover, the smoother eliminates the bias effect along the track observed with the filter on one-message locations due to the use of a random walk dynamics in the model set. The users can take advantage of this new approach through a dedicated reprocessing service made available on the official Argos website (www.argos-system.org). This service is able to deliver upon request and independently of the real-time processing the smoothed estimates for a list of platforms and their associated tracking periods since January 1 st 2008.

Platform terminal transmitters with a limited emitting power or operating in difficult environmental conditions, like wildlife tracking applications, markedly benefit of this new approach. The Argos community has always been extremely prolific and ingenious to develop tools detecting abnormal locations or correcting tracks of this kind of PTTs (see for example [24-29] among many others). These methods are particularly efficient for locations computed with very few messages where the accuracy is limited. The aim of this new processing is not only to improve the overall location accuracy but also to simplify the posterior analysis conducted by the Argos users and to limit the use of multiple post-processing tools. 


\section{Authors' contributions}

All authors contributed equally to the design, conduct, and analysis of this study and to the preparation of this manuscript. All authors read and approved the final manuscript.

\section{Author details}

${ }^{1}$ Collecte Localisation Satellites, 11 rue Hermès, 31520 Ramonville Saint-Agne, France. ${ }^{2}$ CNRS, LAAS, 7 Avenue du colonel Roche, 31400 Toulouse, France. ${ }^{3}$ Univ de Toulouse, UPS, LAAS, 7 Avenue du colonel Roche, 31400 Toulouse, France.

\section{Acknowledgements}

The authors would like to thank all Argos users who kindly provided access to their data so as to qualify the smoothing algorithm presented here. The GPS drifters that provided the data used in the validation analysis were funded by Office of Naval Research Grant N00014-08-1-0557 and by National Oceanic and Atmospheric Administration Grant NA100AR4320156.

All data displayed in this paper remain the property of the different scientists/laboratories listed in Table 1. They provided their Argos/GPS data to the authors for the sole purpose of this paper. People wishing to access these data shall first request them from their data owners. CLS will then gladly provide reprocessed data featured in this paper given the agreement of their owners.

\section{Compliance with ethical guidelines}

\section{Competing interests}

Rémy Lopez, Jean-Pierre Malardé and Philippe Gaspar are employees of Collecte Localisation Satellites which operates the Argos System.

Received: 3 December 2014 Accepted: 11 September 2015 Published online: 29 September 2015

\section{References}

1. Lopez R, Malarde J-P, Royer F, Gaspar P. Improving Argos Doppler location using multiple-model Kalman filtering. IEEE Trans Geosci Remote Sens. 2014;52:4744-55.

2. Blom HAP, Bar-Shalom Y. The interacting multiple model algorithm for systems with Markovian switching coefficients. IEEE Trans Autom Control. 1988;33:780-3.

3. Kalman RE. A new approach to linear filtering and prediction problems. J Basic Eng. 1960;82(Series D):35-45.

4. Wan EA, Van Der Merwe R. The unscented Kalman filter for nonlinear estimation. In: IEEE symposium on adaptive systems for signal communications, and control (AS-SPCC'2000); 2000. p. 153-8.

5. Anderson BDO, Moore JB. Optimal filtering. Courier Corporation; 2012.

6. Li XR, Jilkov VP. Survey of maneuvering target tracking. Part V: multiplemodel methods. IEEE Trans Aerosp Electron Syst. 2005;41:1255-321.

7. Gustafsson F. Adaptive filtering and change detection. New York: Wiley; 2000.

8. Fraser D, Potter J. The optimum linear smoother as a combination of two optimum linear filters. IEEE Trans Autom Control. 1969;14:387-90.

9. Rauch HE, Tung F, Striebel CT. Maximum likelihood estimates of linear dynamic systems. AIAA J. 1965;3:1445-50.

10. Helmick RE, Blair WD, Hoffman SA. Fixed-interval smoothing for Markovian switching systems. IEEE Trans Inf Theory. 1995;41:1845-55.

11. Barber D. Expectation correction for smoothing in switching linear Gaussian state space models. J Mach Learn Res. 2005;7:2515-40.

12. Mesot B, Barber D. A simple alternative derivation of the expectation correction algorithm. IEEE Signal Process Lett. 2009;16:121-4.

13. Barber D. Bayesian reasoning and machine learning. Cambridge: Cambridge University Press; 2012.
14. Ackerson G, Fu K. On state estimation in switching environments. IEEE Trans Autom Control. 1970;15:10-7.

15. Koch W. Fixed-interval retrodiction approach to Bayesian IMM-MHT for maneuvering multiple targets. IEEE Trans Aerosp Electron Syst. 2000;36:2-14.

16. Lopez R, Danès P. Exploiting Rauch-Tung-Striebel formulae for IMMbased smoothing of Markovian switching systems. In: 2012 IEEE international conference on acoustics, speech and signal processing (ICASSP); 2012. p. 3953-6.

17. Lopez R, Danès $P$. A fixed-interval smoother with reduced complexity for jump Markov nonlinear systems. In: 2014 17th international conference on information fusion (FUSION); 2014. p. 1-8.

18. Hawkes LA, Balachandran S, Batbayar N, Butler PJ, Frappell PB, Milsom WK, Tseveenmyadag N, Newman SH, Scott GR, Sathiyaselvam P, Takekawa JY, Wikelski M, Bishop CM. The trans-Himalayan flights of bar-headed geese (Anser indicus). PNAS. 2011;108:9516-9.

19. Benhamou S, Sudre J, Bourjea J, Ciccione S, De Santis A, Luschi P. The role of geomagnetic cues in green turtle open sea navigation. PLoS One. 2011;6:e26672.

20. Villegas-Amtmann S, Simmons SE, Kuhn CE, Huckstadt LA, Costa DP. Latitudinal range influences the seasonal variation in the foraging behavior of marine top predators. PLoS One. 2011;6:e23166.

21. Robinson PW, Costa DP, Crocker DE, Gallo-Reynoso JP, Champagne CD, Fowler MA, Goetsch C, Goetz KT, Hassrick JL, Hückstädt LA, Kuhn CE, Maresh JL, Maxwell SM, McDonald BI, Peterson SH, Simmons SE, Teutschel NM, Villegas-Amtmann S, Yoda K. Foraging behavior and success of a mesopelagic predator in the Northeast Pacific Ocean: insights from a data-rich species, the Northern Elephant Seal. PLoS One. 2012;7:e36728.

22. Dragon A-C, Monestiez P, Bar-Hen A, Guinet C. Linking foraging behaviour to physical oceanographic structures: southern elephant seals and mesoscale eddies east of Kerguelen Islands. Prog Oceanogr. 2010;87:61-71.

23. Dragon A, BarHen A, Monestiez P, Guinet C. Horizontal and vertical movements as predictors of foraging success in a marine predator. Mar Ecol Prog Ser. 2012;447:243-57.

24. Douglas DC, Weinzierl R, Davidson SC, Kays R, Wikelski M, Bohrer G. Moderating Argos location errors in animal tracking data. Methods Ecol Evol. 2012;3(6):999-1007. doi:10.1111/j.2041-210X.2012.00245.X.

25. Tremblay Y, Shaffer SA, Fowler SL, Kuhn CE, McDonald BI, Weise MJ, Bost C-A, Weimerskirch H, Crocker DE, Goebel ME, Costa DP. Interpolation of animal tracking data in a fluid environment. J Exp Biol. 2006;209:128-40.

26. Royer F, Lutcavage M. Filtering and interpreting location errors in satellite telemetry of marine animals. J Exp Mar Biol Ecol. 2008;359:1-10.

27. Jonsen ID, Flemming JM, Myers RA. Robust state-space modeling of animal movement data. Ecology. 2005;86:2874-80.

28. McConnell BJ, Chambers C, Nicholas KS, Fedak MA. Satellite tracking of grey seals (Halichoerus grypus). J Zool. 1992;226:271-82.

29. Austin D, McMillan JI, Bowen WD. A three-stage algorithm for filtering erroneous Argos satellite locations. Mar Mamm Sci. 2003;19:371-83.

\section{Submit your next manuscript to BioMed Central and take full advantage of:}

- Convenient online submission

- Thorough peer review

- No space constraints or color figure charges

- Immediate publication on acceptance

- Inclusion in PubMed, CAS, Scopus and Google Scholar

- Research which is freely available for redistribution

Submit your manuscript at

www.biomedcentral.com/submit 